\title{
Wavelength scaling of terahertz pulse energies delivered by two-color air plasmas
}

Nguyen, A.; Kaltenecker, K. J.; Delagnes, J. C.; Zhou, B.; Cormier, E.; Fedorov, N.; Bouillaud, R.; Descamps, D.; Thiele, I.; Skupin, S.

Total number of authors:

12

Published in:

Optics Letters

Link to article, DOI:

10.1364/OL.44.001488

Publication date:

2019

Document Version

Peer reviewed version

Link back to DTU Orbit

Citation (APA):

Nguyen, A., Kaltenecker, K. J., Delagnes, J. C., Zhou, B., Cormier, E., Fedorov, N., Bouillaud, R., Descamps, D., Thiele, I., Skupin, S., Jepsen, P. U., \& Bergé, L. (2019). Wavelength scaling of terahertz pulse energies delivered by two-color air plasmas. Optics Letters, 44(6), 1488-1491. https://doi.org/10.1364/OL.44.001488

\section{General rights}

Copyright and moral rights for the publications made accessible in the public portal are retained by the authors and/or other copyright owners and it is a condition of accessing publications that users recognise and abide by the legal requirements associated with these rights.

- Users may download and print one copy of any publication from the public portal for the purpose of private study or research.

- You may not further distribute the material or use it for any profit-making activity or commercial gain

- You may freely distribute the URL identifying the publication in the public portal 


\title{
Wavelength scaling of terahertz pulse energies delivered by two-color air plasmas
}

\author{
A. Nguyen ${ }^{1, *}$, K. J. Kaltenecker ${ }^{2}$, J.-C. Delagnes ${ }^{3}$, B. Zhou², E. CormieR ${ }^{3}$, N. Fedorov ${ }^{3}$, \\ R. Bouillaud ${ }^{3}$, D. Descamps ${ }^{3}$, I. Thiele ${ }^{4}$, S. SKupin ${ }^{5}$, P. U. JePsen ${ }^{2}$, AND L. Bergé ${ }^{1}$ \\ ${ }^{1}$ CEA, DAM, DIF - 91297 Arpajon, France \\ ${ }^{2}$ DTU Fotonik, Department of Photonics Engineering, Technical University of Denmark, DK-2800 Kongens Lyngby, Denmark \\ ${ }^{3}$ Univ. Bordeaux - CNRS - CEA, Centre Lasers Intenses et Applications, UMR 5107, 33405 Talence, France \\ ${ }^{4}$ Department of Physics, Chalmers University of Technology, SE-412 96 Göteborg, Sweden \\ ${ }^{5}$ Institut Lumière Matière, UMR 5306 Université Lyon 1 - CNRS, Université de Lyon, 69622 Villeurbanne, France \\ *Corresponding author: luc.berge@cea.fr
}

Compiled January 24, 2019

We address the long-standing problem of the anomalous growth observed in the terahertz $(\mathrm{THz})$ energy yield from air plasmas created by two-color laser pulses, as the fundamental wavelength $\lambda_{0}$ is increased. Using two distinct optical parametric amplifiers (OPA), we report $\mathrm{THz}$ energies scaling like $\lambda_{0}^{\alpha}$ with large exponents $5.6 \leq \alpha \leq 14.3$, which departs from the gfowth in $\lambda$ expected from the photocurrent theory. By means of comprehensive $3 \mathrm{D}$ simulations, we demonstrate that the changes in the laser beam size, pulse duration and phase matching conditions in the second harmonic gen-eration process when tuning the OPA's carrier wave-length can lead to these high scaling powers. The value of the phase angle between the two colors reached at the exit of the doubling crystal turns out to be crucial and even explains non-monotonic behaviors in the mea-surements. (-) 2019 Optical Society of America

OCIS codes: (320.7110) Ultrafast nonlinear optics; (350.5400) Plasmas; (260.3090) Infrared, far.

\section{http://dx.doi.org/10.1364/XX.XX.XXXXXX}

The production of terahertz $(\mathrm{THz})$ radiation by ultrashort laser pulses has become an active field of research because of its promising applications in, e.g., spectroscopy and medical imaging [1]. Efficient $\mathrm{THz}$ emitters can be obtained by focusing into air a two-color femtosecond light pulse, composed of fundamental $(\mathrm{FH})$ and second $(\mathrm{SH})$ harmonics, in order to create a plasma channel that acts as a frequency converter [2]. Temporally asymmetric fields trigger transverse photocurrents through ionization [2], generating broadband $\mathrm{THz}$ pulses.

Recent studies [3-7] showed that increasing the pump wavelength enhances the laser-to-THz conversion efficiency which is usually limited to about $10^{-4}$ for near-infrared (IR) pump pulses. However, there is no consensus achieved on the possible gain factors expected when pushing the $\mathrm{FH}$ wavelength, $\lambda_{0}$, from the near-IR to the mid-IR range. Numerical simulations first displayed a $\mathrm{THz}$ energy increase by a factor 14 when multiplying $\lambda_{0}$ by 2.5 in argon at higher pressure [8]. The seminal paper by Clerici et al. [3] experimentally reported $\mathrm{THz}$ energy yields in air scaling like $\lambda_{0}^{4.6}$ in the range $0.8-1.8 \mu \mathrm{m}$. Later, the local-current model [9] emphasized the crucial role of the relative phase [4], i.e., the largest $\mathrm{THz}$ energy attained by focused two colors with $\pi / 2$ relative phase should follow a scaling in $\lambda_{0}^{2}$ only. A recent work [10] reported a $\mathrm{THz}$ conversion efficiency from noble gases increased by about one order of magnitude when passing from 0.8 to $1.5 \mu \mathrm{m}$. It confirmed the key role of the phase slippage and underlined the existence of an "anomalous" loss of THz energy occurring at specific wavelengths.

Knowing the great sensitivity of $\mathrm{THz}$ emitters with respect to the interaction conditions, we can wonder whether the seemingly contradiction between a $\lambda^{2}$ scaling expected from photocurrents and the steeper increase reported in $[3,10]$ follows from inconsistencies in the beam spatial diameters and pulse durations that may vary a lot in OPAs [11]. Moreover, the relative phase between $\mathrm{FH}$ and $\mathrm{SH}$ fields has a strong impact on the $\mathrm{THz}$ energy [2]. It cannot be directly monitored in experiments, but may change the $\mathrm{THz}$ yield by half an order of magnitude (see, e.g., Fig. 5 of [4]). Thus, revisiting the wavelength scaling of the $\mathrm{THz}$ energy while keeping an eye on both the laser parameters and the value of this phase offset appears timely.

In this Letter we discuss two series of experiments employing distinct TOPAS systems in the wavelength range $1.2-2.6 \mu \mathrm{m}$. Depending on the bandwidth, increases in the THz energy fit a scaling law $\sim \lambda_{0}^{\alpha}$ with large exponents $\alpha$ reaching, e.g., up to 15 between 2.4 and $2.6 \mu \mathrm{m}$. We show that such impressive growths can be explained by the changes in the laser parameters as the OPA wavelength is increased. Comprehensive simulations combining both a unidirectional numerical solver [12] and computations of the $\mathrm{SH} / \mathrm{FH}$ energy ratio from the doubling crystal are performed using experimental beam diameters and pulse durations. They reproduce reasonably well the behaviors of $\mathrm{THz}$ energies reported from the experiments, that is, not only a global growth in $\sim \lambda_{0}^{\alpha}$, but also the occurrence of optimum $\mathrm{FH}$ wavelengths beyond which efficiency in $\mathrm{THz}$ generation drops.

Two experimental campaigns have been performed using two different OPA systems (TOPAS, Light Conversion Ltd). Their 


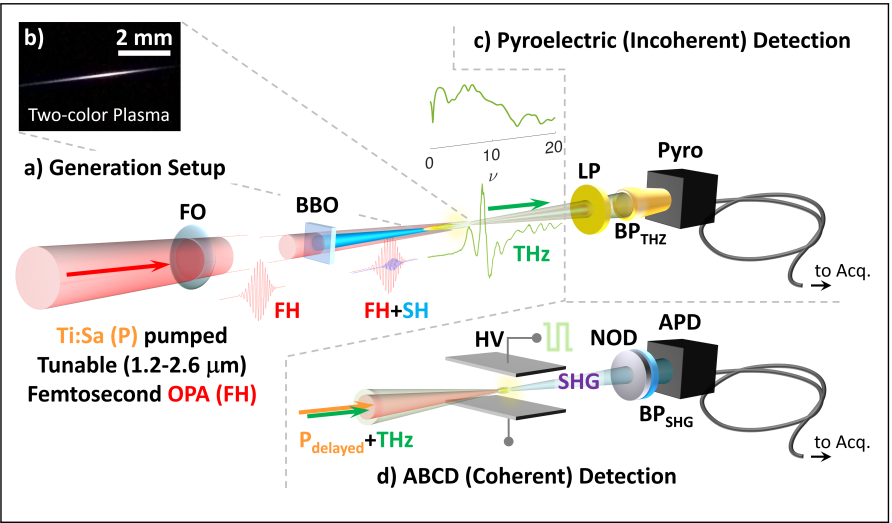

Fig. 1. Sketch for a two-color-driven $\mathrm{THz}$ generation and detection systems. (a) Generation setup. FH goes across focusing optics (lenses or off-axis parabolas, $f=20 \mathrm{~cm}$ ) and the BBO crystal. A 150-fs $\mathrm{THz}$ pulse with spectrum plotted on top is emitted in air by (b) the 5-mm long plasma generated by a 1.5$\mu \mathrm{m}$ FH pulse. (c) Energy measurements using a long-pass filter [LP: teflon tape with a metallic mesh low-pass filter $(20 \mathrm{THz}$, QMC Instruments)] and a band-pass filter (BP) before the pyroelectric (Pyro) detector. (d) ABCD detection system capturing the second harmonic from the delay line coupled with the THz field and a high voltage (HV) bias electric field. The SHG pulse is created by four-wave mixing in air and collected by a neutral optical density (NOD) and a BP filter before reaching an avalanche photodiode (APD). To Acq. : To acquisition.

signal $\left(\lambda_{0} \leq 1.6 \mu \mathrm{m}\right)$ and idler beams $\left(\lambda_{0}>1.6 \mu \mathrm{m}\right)$ were used for plasma generation, while the $800-\mathrm{nm}$ Ti:Sa pulse was exploited in a delay line to measure the $\mathrm{THz}$ electric field.

1/ At DTU Fotonik, a Spectra Physics Solstice Ace laser supplied the 800-nm pump with FWHM duration of $100 \mathrm{fs}$. The OPA (HE-TOPAS Prime) was pumped by $6 \mathrm{~mJ}$ pulses at $1 \mathrm{kHz}$ repetition rate to generate up to $3 \mathrm{~mJ}$ energy beams focused by a lens of $20-\mathrm{cm}$ focal length. The FH pulse was sent into a 100$\mu$ m-thick $\beta$-barium borate (BBO) crystal for second harmonic generation (SHG). Off-axis parabolic mirrors collimated the $\mathrm{THz}$ field. A combination of first a Teflon tape and then a metallic mesh low-pass filter was employed to block any residual $\mathrm{FH}$ and $\mathrm{SH}$ components. Detection of the $\mathrm{THz}$ field was done by using the air-biased coherent detection (ABCD) technique [13]. An avalanche photodiode (APD) [14] served as photodetector and a pyroelectric detector (QMC Instruments Ltd) performed the energy measurements in the frequency window $v<20 \mathrm{THz}$ at constant $\mathrm{FH}$ power in the ranges $1.2-1.5 \mu \mathrm{m}$ and $2.4-2.6 \mu \mathrm{m}$.

2/ At CELIA, the TOPAS system was pumped by a Ti:Sa amplifier ( $1 \mathrm{k} \mathrm{Hz}, 25 \mathrm{fs}, 4.5 \mathrm{~mJ})$ and could d eliver, e.g., $2-\mu \mathrm{m}$ pulses with 60 -fs FWHM duration. Basically the same setup as in DTU Fotonik was exploited. The same pyroelectric detector was used for measuring $\mathrm{THz}$ energy yields continuously in the wavelength domain $1.3-2.2 \mu \mathrm{m}$ for a beam energy adjusted between 0.1 and $0.6 \mathrm{~mJ}$. A sketch of the experimental setup showing a 5-mm long plasma trace and a typical $\mathrm{THz}$ waveform is illustrated in Fig. 1. Apart from their amplitude value, the spectral shapes did not significantly vary when increasing $\lambda_{0}$ compared with $\mathrm{THz}$ spectra reported in the literature [3].

Figure 2 summarizes the THz signals recorded in the two experimental campaigns. Despite the different wavelength ranges investigated, the fitting curves of this figure underlines impres-

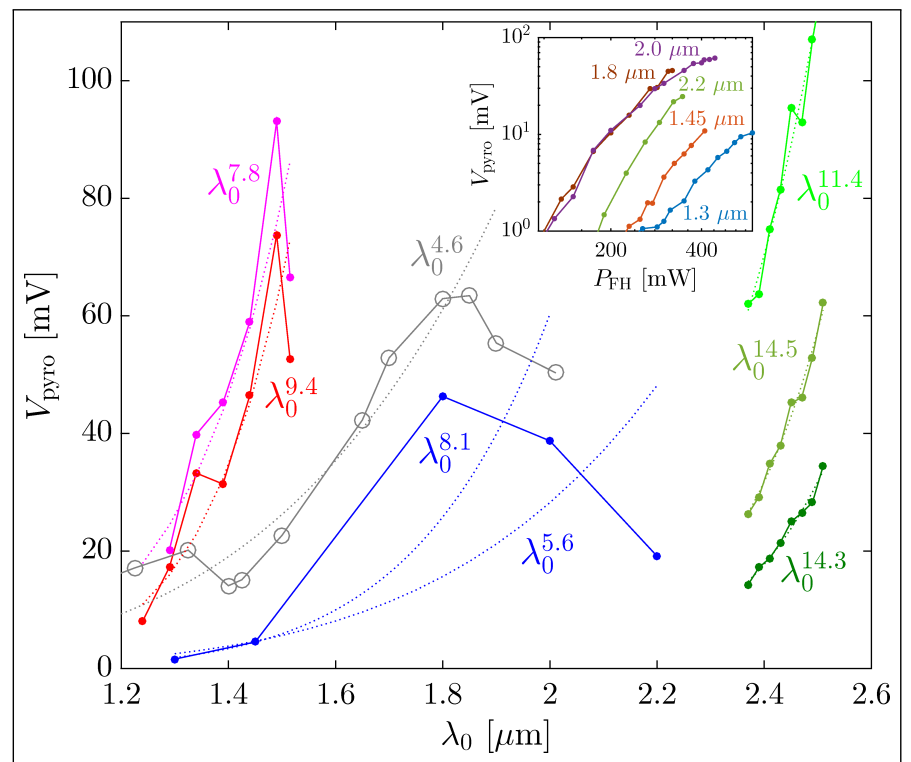

Fig. 2. Signal of the pyroelectric detector supplying the energy yield vs $\lambda_{0}$ in the frequency window $v<20 \mathrm{THz}$. DTU measurements (red/pink curves: $1.2-1.5 \mu \mathrm{m}$ and green curves: $2.4-2.6 \mu \mathrm{m}$ ) lie aside the CELIA ones (blue curve: $1.3-2.2$ $\mu \mathrm{m})$. The average input power of the pump beam is fixed for each interval of scanned wavelength, namely, $840 \mathrm{~mW}$ (pink), $720 \mathrm{~mW}$ (red), $300 \mathrm{~mW}$ (blue), $348 \mathrm{~mW}$ (dark green), $420 \mathrm{~mW}$ (middle green) and $558 \mathrm{~mW}$ (light green curve). Dotted curves are fitting curves in $\lambda^{\alpha}$ (with and without the last point included when fitting the blue curve). Gray circles recall the data of Ref. [3]. Inset shows the Pyro signal varying with the $\mathrm{FH}$ averaged power for the CELIA experiment.

sive growths in $\lambda^{\alpha}$ with powers $\alpha$ not only exceeding $\sim 4.6$ [3], but also reaching $\alpha \approx 7-9$, up to almost 15 in the highest wavelength ranges. Note, however, that the largest exponents refer to the narrowest bandwidths in FH wavelengths. Because such a narrow wavelength range naturally limits the retrieval of an accurate scaling law, we shall henceforth focus our analysis on the lower FH wavelengths. In the broadest range $1.3 \leq \lambda_{0} \leq 2.2 \mu \mathrm{m}$, the slope in the THz energy gains remain closer to Clerici's data (gray symbols). Concatenation of our data points for $\lambda_{0} \leq 2.2$ $\mu$ m provides a global scaling in power $\alpha \approx 7.7$ that differs from the basic $\lambda^{2}$ scaling. We can also notice the decrease in the pyroelectric signal occurring beyond specific $\lambda_{0} \mathrm{v}$ alues. So, the curves of Fig. 2 mainly exhibit a generic growth in $\lambda^{\alpha}$, which can be stopped at some optimum wavelengths where maximum $\mathrm{THz}$ generation is locally reached.

Let us now discuss the influence of the various laser parameters. For the sake of clarity, these can be classified into two categories, namely, the envelope laser parameters such as the beam width, pulse duration, $\mathrm{SH} / \mathrm{FH}$ energy ratio, and the phase angle between the two colors. Figures $3(a, b)$ detail the pump beam diameter and pulse durations reported from the DTU's TOPAS, measured with a scanning-slit beam profiler. These subplots, in agreement with [11], display evidence that both pulse initial width and duration can evolve with $\lambda_{0}$ by a couple of $\mathrm{mm}$ or tens of fs in the $1.3-1.8 \mu \mathrm{m}$ range, respectively. In our experiments the BBO crystal was positioned at a fixed distance of $5 \mathrm{~cm}$ from the linear focus, supplying sufficient SH energy while avoiding crystal damage. Typically an $\mathrm{SH} / \mathrm{FH}$ energy ratio of 


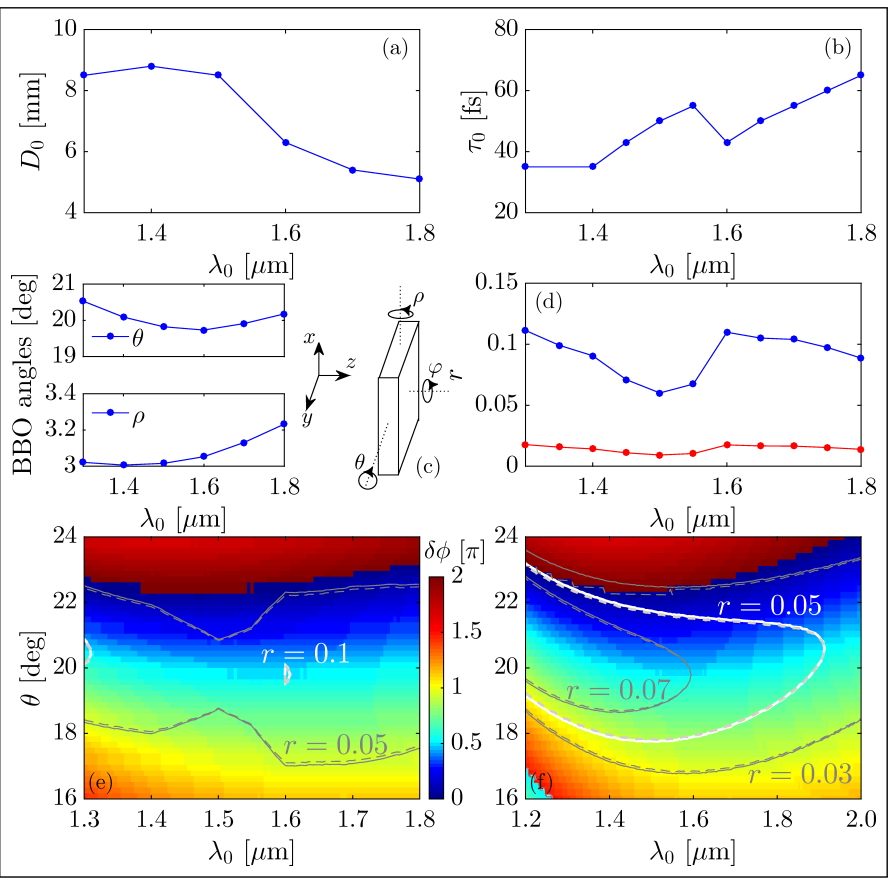

Fig. 3. (a) Output FWHM diameters and (b) pulse durations as function of the FH wavelength measured from the DTU TOPAS. Error bars (not shown) are $\pm 10 \%$. (c) Variations of angles $\theta$ and $\rho$ optimizing SHG through pump polarization and temporal walk-off across the BBO [15]. (d) $\lambda_{0}$-dependent variations of the $\mathrm{SH} / \mathrm{FH}$ energy ratio $r$ computed from Eq. (1) for a $100-\mu \mathrm{m}$ thick BBO crystal, accounting for variations in $D_{0}$ and $\tau_{0}$ setting $\delta k=0$. Blue curve: $\varphi=0$. Red curve: projected $\mathrm{SH}$ with $\varphi=0.2 \pi$ (see text). (e,f) Relative phase $\delta \phi$ between the two colors induced by the BBO crystal varying with $\left(\theta, \lambda_{0}\right)$ in (e) CELIA $(\varphi=0.2 \pi)$ and (f) Clerici configurations ( $\varphi=$ $0.4 \pi$ ). Lines are $r$-levels calculated with the SHG model (solid) or fitted with $r \propto \operatorname{sinc}^{2}(\delta \phi-\pi / 2)$ (dashed lines).

$\sim 10-12 \%$ was measured for $1.3 \leq \lambda_{0} \leq 2.2 \mu \mathrm{m}$. Changes in these parameters affect the $\mathrm{SH} / \mathrm{FH}$ performances of the $\mathrm{BBO}$ crystal and the local intensity reached at focus, both impacting the $\mathrm{THz}$ conversion mechanism. Our data encompass variations in both classes of laser parameters, which explains the variable growths in the THz yields. When $\lambda_{0}$ is increased, enhancing the pulse duration increases the number of ionization events, which contribute constructively to the photocurrent-induced $\mathrm{THz}$ field [8]. The decrease in the beam spatial width favors higher initial intensities, which trigger plasma generation earlier along the optical path and accumulates more $\mathrm{THz}$ energy. For comparison, the pulse duration of Ref. [3] was maintained within a fine margin of $\pm 5 \mathrm{fs}$ and the $\mathrm{SH} / \mathrm{FH}$ energy ratio was kept inside the narrow range of $5 \pm 2 \%$. A constant beam diameter was conjectured [16] and the BBO-plasma distance was $\approx 1.2 \mathrm{~cm}$. Thus, because the envelope parameters were kept quasi-constant here, the $\mathrm{THz}$ yield should be mainly sensitive to phase variations. In all experiments the basic protocol was the same: first, the BBO-focus distance was fixed at the lowest FH wavelength of a given scanned frequency interval and the $\mathrm{BBO}$ angles were varied to collect maximum $\mathrm{THz}$ energy. Thus, the $\mathrm{FH}$ polarization was rotated with respect to the crystal's ordinary axis ( $\mathrm{SH}$ is produced on the orthogonal extraordinary axis), in order to find a compromise between producing a relevant (but not maximum) amount of SH and having a large enough $\mathrm{SH}$ vector component parallel to the $\mathrm{FH}$ polarization direction for an efficient $\mathrm{THz}$ generation. Second, keeping the same BBO-focus distance, maximum $\mathrm{THz}$ energy was sought for higher $\mathrm{FH}$ wavelengths by only manipulating the angles of the BBO crystal.

Our numerical approach carefully followed the experimental protocol. The relevant SHG quantities, i.e., the SH/FH energy ratio $r$ and phase angle between the two colors $\delta \phi$, were obtained through a standard $\chi^{(2)}$ envelope model [17]:

$$
\partial_{z} \widehat{A_{2}}=\mathrm{i} \frac{\omega_{2} \chi^{(2)}}{c n_{c, 2}} \widehat{A_{1}^{2}} \mathrm{e}^{\mathrm{i} \delta k z}, \partial_{z} \widehat{A_{1}}=\mathrm{i} \frac{\omega_{1} \chi^{(2)}}{c n_{c, 1}} \widehat{A_{1}^{*} A_{2}} \mathrm{e}^{-\mathrm{i} \delta k z}
$$

where index $j=1,2$ refers to the $\mathrm{FH}$ and $\mathrm{SH}$ components, respectively attached to the slowly-varying envelopes $A_{j}$ of the $j$ th color field $\widehat{E}_{j}\left(\omega_{j}, z\right)=\widehat{A_{j}}(\omega, z) \exp \left[\mathrm{ij} n_{c, j}(0) \omega_{0} z / c\right]$ at frequencies $\omega_{j}=\omega+j \omega_{0}$. The $\omega$-dependent refraction index $n_{c, j}(\omega)$ is the BBO dispersion index changing along the ordinary $\left[n_{c, 1}(\omega)=n_{o}\left(\omega_{1}\right)\right]$ and extraordinary $\left[n_{c, 2}(\omega)=n_{e}\left(\theta, \omega_{2}\right)\right]$ axes, while $\delta k(\omega) \equiv\left(n_{c, 1}-n_{c, 2}\right) \omega_{2} / c$ is the phase mismatch in wavenumber. First, we checked that the optimum values for polar and azimuthal angles of the BBO crystal assuring maximum SHG do not vary so much in the scanned range of wavelengths [see Fig. 3(c)]. Second, we evaluated the local FH intensity deposited on the doubling crystal using the BBO positions, beam diameters and energies (powers) mentioned above for extracting the corresponding $\mathrm{SH} / \mathrm{FH}$ ratios from Eq. (1). The resulting SHG energy ratio $r$ is shown by the solid blue curve in Fig. 3(d) and remains consistent with the experimental values. The $\mathrm{BBO}$ angle $\varphi$ has a strong impact on $r$, because the $\mathrm{FH}$ has to be projected on the ordinary polarization axis, so that $r(\varphi)=r(\varphi=0) \cos ^{2}(\varphi)$. Completing the BBO response, Figs. $3(\mathrm{e}, \mathrm{f})$ detail the phase angle $\delta \phi$ of the two-color pulse at the exit of the BBO crystal versus $\lambda_{0}$ and the azimuthal angle $\theta$. It is possible to extract from Eq. (1) in the undepleted pump approximation the relationships $r \propto \operatorname{sinc}^{2}(\delta k L / 2) ; \delta \phi=\pi / 2+\delta k L / 2$ with $L=100 \mu \mathrm{m}$ denoting the BBO thickness and $\operatorname{sinc}(x)=\sin (x) / x$. Results from this analytical evaluation employing $\delta k(\theta, \omega)$ are plotted as dashed lines in Figs. 3(e,f) and they superimpose with the full numerical solutions of Eq. (1). The $\delta \phi$ values cover almost a $\pi$ interval when $r$ is halved. Therefore, there is some uncertainty in the values of the phase angle when one tries to reproduce the experimental results.

Next, we numerically solved the standard scalar 3D unidirectional pulse propagation equation (UPPE) $[12,18]$

$$
\partial_{z} \hat{E}=\mathrm{i} \sqrt{k^{2}(\omega)-k_{x}^{2}-k_{y}^{2}} \hat{E}+\mathrm{i} \frac{\mu_{0} \omega^{2}}{2 k(\omega)} \hat{\mathcal{F}}_{\mathrm{NL}},
$$

which describes linear dispersion and diffraction of the pulse electric field $\hat{E}\left(k_{x}, k_{y}, z, \omega\right)$ in Fourier domain, as well as the nonlinear polarization, the electron current and ionization

losses gathered in the nonlinear response $\hat{\mathcal{F}}_{\mathrm{NL}}$ (see [4] for details related to the physical constants). The input two-color laser field is Gaussian, both in space and time, with a factor $\exp \left[\mathrm{i} \omega\left(x^{2}+y^{2}\right) / 2 c f\right]$ [19] accounting for the focal length $f$. For practical uses of this scalar UPPE model, the effective SH input intensity was decreased by an additional factor $\sim \sin ^{2} \varphi$ due to projection on the FH polarization axis. The $r$ ratios used in the simulations are specified by the red curve in Fig. 3(d).

We first simulated the growth in the $\mathrm{THz}$ y ields reported in Ref. [3]. The solid red curve of Fig. 4(a) nicely reproduces Clerici et al.'s experimental results for an $\mathrm{SH} / \mathrm{FH}$ ratio equal to $5 \pm 2 \%$ and $\varphi=0.4 \pi$. The relative phase $\delta \phi$ introduced by the 

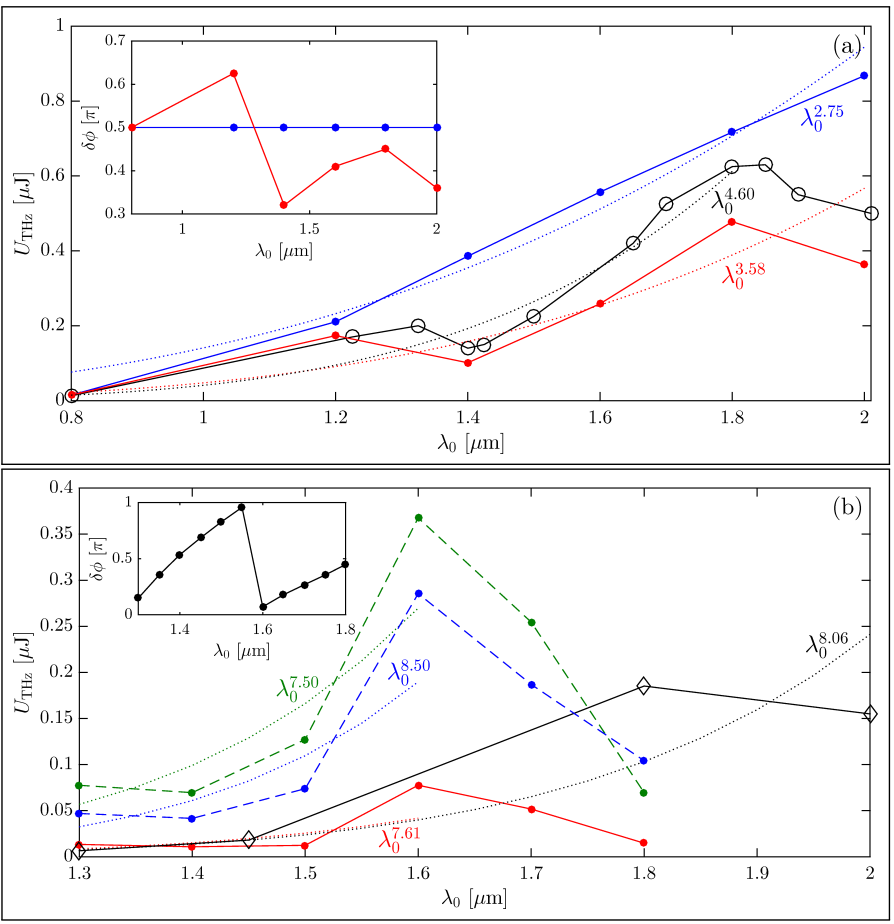

Fig. 4. (a) THz energy yield computed with the 3D UPPE model (red dots) for the experimental data of [3] (black circles) with the phase shifts $\delta \phi$ specified in inset employed for a BBO crystal located at $1.2 \mathrm{~cm}$ from focus. The blue curve is associated to $\delta \phi=\pi / 2$ at the exit of the BBO crystal. (b) THz yield simulated for a BBO crystal at $5 \mathrm{~cm}$ prior to focus computed from the relative phase $\delta \phi$ for $300 \mu \mathrm{J}$ (red) and $800 \mu \mathrm{J}$ (blue curves) pump pulses. The black curve displays the experimental signals converted in $\mu \mathrm{J}$ for $1 \mathrm{kHz}$ modulation frequency and a response function of $250 \mathrm{~V} / \mathrm{W}$. The green curve shows the $\mathrm{THz}$ energy yield using $5 \% \mathrm{SH} / \mathrm{FH}$ energy ratio for a $300 \mu \mathrm{J}$ pump pulse. Dotted lines display fitting curves.

BBO crystal and selected consistently with Fig. 3(f) for each FH wavelength is shown in the inset. Here, the uncertainty in $\delta \phi$ mentioned above enables us to choose values in the allowed interval bounded by the gray curves in Fig. 3(e), in order to match the experimental curve. Drops in the THz signal beyond specific wavelengths $\left(\lambda_{0}=1.4\right.$ and $\left.2.0 \mu \mathrm{m}\right)$ are due to variations in $\delta \phi$, leading to strong phase shift at focus from the optimal phase angle $\pi / 2$ that maximizes the THz energy by photocurrents. Higher THz generation is indeed reached whenever $\delta \phi=\pi / 2$ (blue curve), which corresponds to a perfect phase-matching in the BBO crystal. The scaling evaluated with this phase angle is $\lambda_{0}^{2.75}$ for $\lambda_{0} \geq 1.2 \mu \mathrm{m}$, which agrees with the estimates of [4] The data point at $800 \mathrm{~nm}$, however, lies outside this curve, because the phase shift achieved inside the plasma is further from $\pi / 2$ due to, e.g., stronger dispersion. Next, we focused on the data of Fig. 2 in the range $1.3-1.8 \mu \mathrm{m}$, using the beam widths and pulse lengths of Figs. 3(a,b) and the effective $\mathrm{SH} / \mathrm{FH}$ ratios $r$ of Fig. 3(d) for $\varphi=0.2 \pi$. The selected phase angles, again consistent with Fig. 3(e), are displayed in the inset. However, this time we did not try to match the experimental curve, but chose an almost linear distribution, with a $\pi$-jump between 1.5 and $1.6 \mu \mathrm{m}$. Figure 4 (b) shows the resulting energy growths computed at the fixed pump energies of $300 \mu \mathrm{J}$ (red) and $800 \mu \mathrm{J}$ (blue curves), representative of the CELIA and DTU setups. For comparison, the green curve shows the $\mathrm{THz}$ yield produced with a constant $\mathrm{SH} / \mathrm{FH}$ ratio of $r=5 \%$. All THz yields show an increase at $\lambda_{0}=1.6 \mu \mathrm{m}$, fitting a $\sin ^{2}\left(\delta \phi+\phi_{p}\right)$ law, where $\phi_{p}$ $\simeq 0.45 \pi$ is an almost constant phase shift due to linear and nonlinear propagation. Hence, our simulations allow us to attribute the sudden changes in the $\mathrm{THz}$ yield to variations of the phase angle between the two colors. From these results we infer that the impressive scaling laws reported in the experiments can be linked to variations in the initial beam size and pulse duration induced by the OPA, and to the lack of control of the phase angle $\delta \phi$ at the BBO exit.

In conclusion, our analysis highlighted the critical dependencies of the measured $\mathrm{THz}$ yield against the OPA beam parameter and $\mathrm{SH}$ generation in the BBO. Our numerical and experimental data support the best mutual agreement achieved up to date. We show that fluctuations in the pump parameters, particularly in the relative phase between the two colors, can have a tremendous effect on the $\mathrm{THz}$ yield, and may explain various observable scaling laws. Future technological investigations should thus focus on the control of this relative phase and more generally on new OPA architectures immune from distortions in the beam spatial wavefront and pulse duration.

Funding ANR/ASTRID Project "ALTESSE" (ANR-15-ASTR0009); GENCI (A0020507594); Qatar National Research Fund (NPRP 8-246-1-060); ANR/ASTRID Project "ArchiMid" (ANR15-ASTR-0005); Laserlab.dk (Danish Center for Laser Infrastructure); Laserlab-Europe EU-H2020 (654148).

Acknowledgement The authors thank Matteo Clerici for fruitful discussions and Laurent Merzeau for advices in optoelectronics.

\section{REFERENCES}

1. M. Tonouchi, Nature Photon. 1, 97 (2007).

2. K. Y. Kim, A. J. Taylor, J. H. Glownia, and G. Rodriguez, Nature Photon. 2, 605 (2008).

3. M. Clerici, M. Peccianti, B. Schmidt, L. Caspani, M. Shalaby, M. Giguère, A. Lotti, A. Couairon, F. Légaré, T. Ozaki, D. Faccio, and R. Morandotti, Phys. Rev. Lett. 110, 253901 (2013).

4. A. Nguyen, P. González de Alaiza Martínez, J. Déchard, I. Thiele, I. Babushkin, S. Skupin, and L. Bergé, Opt. Express 25, 4720 (2017).

5. V. Y. Fedorov and S. Tzortzakis, Phys. Rev. A 97, 063842 (2018).

6. A. Nguyen, P. González de Alaiza Martínez, I. Thiele, S. Skupin, and L. Bergé, Phys. Rev. A 97, 063839 (2018).

7. V. Y. Fedorov and S. Tzortzakis, Opt. Express 26, 31150 (2018).

8. L. Bergé, S. Skupin, C. Köhler, I. Babushkin, and J. Herrmann, Phys. Rev. Lett. 110, 073901 (2013).

9. I. Babushkin, S. Skupin, A. Husakou, C. Köhler, E. Cabrera-Granado, L. Bergé, and J. Herrmann, New J. Phys. 13, 123029 (2011).

10. H. Zhao, L. Zhang, S. Huang, S. Zhang, and C. Zhang, IEEE THz Sc. Techn. 8, 299 (2018).

11. N. S. Makarov, M. Drobizhev, and A. Rebane, Opt. Express 16, 4029 (2008).

12. M. Kolesik and J. V. Moloney, Phys. Rev. E 70, 036604 (2004).

13. J. Dai, X. Xie, and X.-C. Zhang, Phys. Rev. Lett. 97, 103903 (2006).

14. T. Wang, K. Iwaszczuk, E. A. Wrisberg, E. V. Denning, and P. U. Jepsen, J. Infrared Milli. Terahz. Waves 37, 592 (2016).

15. G. Tamosauškas, G. Beresnevičius, D. Gadonas, and A. Dubietis, Opt. Materials Express 6, 1410 (2018).

16. M. Clerici, Private Communication (2018).

17. R. W. Boyd, ed., Nonlinear Optics (Academic Press, San Diego, 1992).

18. M. Kolesik, J. V. Moloney, and M. Mlejnek, Phys. Rev. Lett. 89, 283902 (2002).

19. L. Bergé, S. Skupin, and G. Steinmeyer, Phys. Rev. A 79, 033838 (2009). 